\title{
BIOLOGIA DA MARIPOSA-ORIENTAL EM DIETA ARTIFICIAL Á BASE DE MILHO
}

\section{BIOLOGY OF THE ORIENTAL FRUIT MOTH IN ARTIFICIAL CORN-BASED DIET}

\author{
Cristiano João ARIOLI ${ }^{1}$ \\ Mauro Silveira GARCIA ${ }^{2}$ \\ Marcelo ZARTE ${ }^{3}$ \\ Marcos BOTTON ${ }^{4}$
}

\section{RESUMO}

A biologia de Grapholita molesta (Busck) foi estudada numa dieta artificial a base de milho comparada com a de Ivaldi-Sender, referência para a criação do inseto em laboratório. $O$ experimento foi conduzido em laboratório sob temperatura de $24 \pm 2{ }^{\circ} \mathrm{C}, 70 \pm 10 \%$ UR e fotofase de 16 horas, comparando-se as dietas através do emprego da tabela de vida de fertilidade. Na dieta a base de milho foi registrada maior duração no período de lagarta-adulto e menor fecundidade total. A dieta à base de milho proporcionou maior tempo de desenvolvimento de uma geração $(T)$, menor taxa líquida de reprodução (Ro), menor capacidade inata de aumentar em número $(\mathrm{rm})$ e menor razão finita de aumento $(\lambda)$. Contudo, a dieta à base de milho também pode ser empregada na criação do inseto, pois além de ser de fácil obtenção e preparo, proporcionou uma viabilidade total do período lagarta-adulto de $87,2 \%$.

Palavras-chave: Grapholita molesta; dieta artificial; tabela de vida de fertilidade, criação massal.

Grapholita molesta (Busck) biology was studied in a corn-based diet compared with Ivaldi-Sender (1976), standard substrate for insect rearing in laboratory. The experiment was conducted in laboratory $\left(24 \pm 2{ }^{\circ} \mathrm{C}, 70 \pm 10 \% \mathrm{RU}\right.$ and photophase of $16 \mathrm{~h}$ ) comparing diets through fertility life table. The corn-based diet showed a longer duration of the larvae to adult stage and a lower fecundity. The corn-based diet provided a shorter generation time $(T)$, a minor net reproductive rate (Ro), a minor innate capacity for increase in numbers $(\mathrm{rm})$ and a minor finite rate of increase (I). However, the corn-based diet can also be used for rearing G. molesta, because beyond being of easy attainment and preparation, provided a total viability for the larvae to adult of $87.2 \%$.

Key-words: Grapholita molesta; artificial diet; fertility life table; mass rearing.

\footnotetext{
${ }^{1}$ Dr. Pesquisador. EPAGRI - Estação Experimental de Videira. Rua João Zardo, 1660, Bairro Campo Experimental. CEP. 89.560-000, Videira, SC, Brasil. E-mail: cristianoarioli@epagri.sc.gov.br. Autor para correspondência.

2 Dr. Professor, Universidade Federal de Pelotas, Departamento de Fitossanidade, Campus Universitário, s/n - CP 354 CEP $96.010-900$ Pelotas, RS, Brasil.

3 Doutorando em Fitotecnia, Universidade Federal do Rio Grande do Sul, Avenida Bento Gonçalves, 7712,CEP. 91540-000. Porto Alegre, RS, Brasil. E-mail: marcelo_zart@yahoo.com.br.

${ }^{4}$ Dr. Pesquisador. Embrapa Uva e Vinho. Rua Livramento, 515, CAP. 130. CEP. 97.500-000, Bento Gonçalves, RS, Brasil. E-mail: marcos@cnpuv.embrapa.br
} 
ARIOLI, C. J. et al. Biologia da mariposa oriental em dieta...

\section{INTRODUÇÃO}

A mariposa-oriental Grapholita molesta (Busck, 1916) (Lepidoptera: Tortricidae) é uma das principais pragas das fruteiras de clima temperado no Brasil, com destaque para a ameixeira, macieira, pereira e pessegueiro (Nora \& Sugiura, 2001; Salles, 2001; Kovaleski \& Ribeiro, 2002; Nora \& Hickel, 2002; Arioli et al., 2003 e 2004; Monteiro \& Hickel, 2004;).

Como forma de desenvolver estratégias eficazes para o manejo integrado da mariposaoriental, a espécie tem sido criada mundialmente utilizando dieta natural ou artificial e em escala experimental e massiva (Ivaldi-Sender, 1974; Szocs \& Tòth, 1982; Yokoyama et al., 1987; Rosenthal et al., 1994; Ribbert \& Marín, 2005; Marín et al., 2006).

No Brasil, mesmo com a importância de $G$. molesta como praga das rosáceas, até 2007 não houve preocupação em implementar uma criação em laboratório de forma a produzir insetos em grandes populações para trabalhos de comportamento e controle. Nos estudos conduzidos até então, os insetos foram criados em pequena escala (Grellmann et al., 1991; Rosenthal et al., 1994) ou coletados diretamente dos pomares comerciais (Arioli et al., 2004). Dessa forma, a partir do estabelecimento de uma metodologia de criação do inseto em laboratório (Arioli et al., 2007) considera-se importante prospectar outros substratos alimentares para emprego em dieta artificial com o objetivo de facilitar a criação do inseto. A dieta considerada referência para a criação da G molesta é a proposta por Ivaldi-Sender (1974), a qual utiliza alimento natural (maçã seca) como um dos ingredientes básicos. Por envolver uma maior manipulação deste ingrediente (o qual deve ser secado e triturado no laboratório) a confecção da dieta acaba exigindo maior tempo e trabalho, além de laboratório com maior infraestrutura. Levando em conta a facilidade na obtenção dos ingredientes bem como a praticidade de elaboração, uma dieta a base de milho (que exclui a componente maçã seca), proposta por Arioli et al. (2007), tem sido amplamente utilizado na maioria dos laboratórios do Brasil que passaram a manter criações de G. molesta. No entanto, não existem informações sobre o efeito deste alimento na biologia da espécie.

Este trabalho teve por objetivo avaliar o desenvolvimento biológico de G. molesta em uma dieta à base de milho em comparação a dieta mundialmente recomendada para a criação da espécie em laboratório.

\section{MATERIAL E MÉTODOS}

O trabalho foi conduzido no Laboratório de Entomologia da Embrapa Uva e Vinho, localizado em Bento Gonçalves, RS, sob temperatura de $24 \pm 2$ ${ }^{\circ} \mathrm{C}$, UR de $70 \pm 10 \%$ e fotofase de $16 \mathrm{~h}$.

\section{Obtenção dos insetos}

Maçãs do cultivar Fuji infestadas com $G$. molesta foram obtidas em fevereiro de 2005, em pomares comercias de Vacaria, RS (latitude $28^{\circ} 26^{\prime}$ 'S e longitude $50^{\circ} 48^{\prime} \mathrm{O}$ ). Após a coleta, as maçãs foram levadas ao laboratório e colocadas em bandejas plásticas $(59 \times 38 \times 8,5 \mathrm{~cm})$ e mantidas em sala climatizada. Sobre estas foram dispostas sete camadas de tecido de gaze $(25 \times 25 \mathrm{~cm})$ que foram distribuídas uma sobre a outra formando uma cobertura uniforme sobre os frutos, permitindo assim a formação e obtenção das pupas. Posteriormente, os insetos foram criados em condições controladas seguindo a metodologia descrita por Arioli et al. (2007). O desenvolvimento e a reprodução de $G$. molesta nas duas dietas artificiais (Tabela 1) foram comparados através das seguintes variáveis biológicas: duração e viabilidade das fases de ovo, lagarta, pupa e do período de lagarta à emergência do adulto (lagarta-adulto); razão sexual; massa de pupas; período de préoviposição, oviposição; fecundidade diária e total e longevidade dos adultos.

TABELA 1 - Composição da dieta padrão (Ivaldi-Sender) e da dieta avaliada (à base de milho) para criação de G. molesta em laboratório e componentes da solução nutritiva utilizada para alimentação de adultos.

\begin{tabular}{lcc}
\hline \multicolumn{1}{c}{ Componentes da dieta para a fase de lagarta ${ }^{1}$} & \multicolumn{2}{c}{ Dieta } \\
\cline { 2 - 3 } & Ivaldi-Sender & À base de milho \\
\hline Farinha de milho & $50,0 \mathrm{~g}$ & $174,0 \mathrm{~g}$ \\
Maçã seca (moída) & $50,0 \mathrm{~g}$ & - \\
Germe de trigo & $50,0 \mathrm{~g}$ & $43,5 \mathrm{~g}$ \\
Levedura de cerveja & $50,0 \mathrm{~g}$ & $46,6 \mathrm{~g}$ \\
Ácido ascórbico & $4,5 \mathrm{~g}$ & $6,2 \mathrm{~g}$ \\
Ácido Benzóico & $1,5 \mathrm{~g}$ & $2,8 \mathrm{~g}$ \\
Metil parahidroxibenzoato (Nipagin) & $1,5 \mathrm{~g}$ & $2,2 \mathrm{~g}$ \\
Formaldeído & - & $0,8 \mathrm{~cm}^{3}$ \\
Ágar & $18,0 \mathrm{~g}$ & $24,8 \mathrm{~g}$ \\
\hline Componentes da dieta para a fase adulta ${ }^{2}$ & - & - \\
\hline Mel & & $130,3 \mathrm{~g}$ \\
Metil parahidroxibenzoato (Nipagin) & \multicolumn{2}{c}{ - } \\
\hline
\end{tabular}

${ }^{1-}$ Com adição de água destilada para completar $1 \mathrm{~kg}$ de dieta/

${ }^{2-}$ Com adição de água destilada para completar $1 \mathrm{dm}^{3}$ de solução. 


\section{Biologia de G. molesta}

Lagartas recém-eclodidas da geração F4 (150 por tratamento) obtidas da criação estoque mantida segundo a metodologia de Arioli et al. (2007) foram transferidas, com o auxílio de um pincel fino, em câmara asséptica, para tubos de vidro $(2,5 \times 8,5 \mathrm{~cm})$, previamente esterilizados, contendo $4 \mathrm{~g}$ de uma das dietas em teste (Tabela 1). Cada tubo recebeu uma lagarta, os quais foram tamponados com algodão hidrófobo para evitar a fuga do inseto e também para servir de local para a formação das pupas. Estas, com até $24 \mathrm{~h}$ de idade, foram pesadas e separadas por sexo, conforme sexagem proposta por Beeke \& Jong (1991). Posteriormente, foram transferidas para tubos de vidro contendo papel-filtro umedecido até a emergência dos adultos.

Para o estudo da fecundidade e longevidade, 20 casais foram individualizados em gaiolas confeccionadas com copos de PVC transparente semiflexível, com capacidade de 200 $\mathrm{cm}^{3}$, nas paredes do qual os adultos da mariposaoriental realizavam a postura. A abertura do copo foi fechada com tecido de voile, preso com o auxílio de um elástico para a circulação e troca de ar com o ambiente externo. A extremidade inferior do copo foi perfurada no centro para a inserção de algodão embebido em solução alimentar para adultos (Tabela1), sendo este substituído diariamente. O número de ovos e a mortalidade dos adultos foram registrados diariamente. Os ovos com 24 a $48 \mathrm{~h}$ foram incubados separadamente em gaiolas de copo de PVC, para avaliação do período de incubação e a viabilidade dos mesmos.

A partir dos dados de biologia, foi calculada a tabela de vida de fertilidade para $G$. molesta com as duas dietas (Silveira Neto et al., 1976), estimando, para as futuras gerações, a capacidade inata de aumentar em número $\left(r_{m}\right)$, o número de vezes que cada espécie pode aumentar
(Ro), o intervalo de tempo entre cada geração $(T)$ e o número de indivíduos adicionados à população, por fêmea, por dia e que darão origem a fêmeas $(\lambda)$.

$\mathrm{O}$ delineamento experimental foi inteiramente casualisado, considerando-se repetição cada lagarta ou cada casal para a avaliação da fecundidade e longevidade. Os dados obtidos foram analisados quanto à normalidade pelo teste de Liliefors e quanto à homogeneidade das variâncias dos erros pelos testes de Cochrane e Bartlett para os experimentos com número igual e diferente de repetições, respectivamente (Ribeiro Júnior, 2001). Assim, os dados (duração dos estágios, massa de pupa, longevidade e fecundidade) que não apresentaram distribuição normal ou homogeneidade da variância foram transformados em $(x+0,5)^{0,5}$ e os dados expressos em porcentagem (viabilidade), transformados em arcseno $(x / 100)^{0,5}$. As médias dos tratamentos foram comparadas pelo teste $\mathrm{T}$ a probabilidade de erro de 0,05 .

\section{RESULTADOS E DISCUSSÃO}

O tempo de desenvolvimento da mariposaoriental foi alterado na dieta à base de milho (Tabelas 2 e 3) em comparação com a dieta padrão. Com exceção da duração na fase de pupa, a qual foi menor nesta dieta ( 8,5 a 8,7 dias), as durações do estágio de lagarta e de lagarta-adulto foram significativamente menores na dieta proposta por Ivaldi-Sender (1974). Os períodos de oviposição, longevidade (machos e fêmeas) e fecundidade total também foram maiores para a dieta de Ivaldi-Sender, indicando ter havido um melhor efeito nutricional da dieta padrão, já que o desempenho foi melhor quando as lagartas se desenvolveram neste meio (Tabelas 3 e 4).

TABELA 2 - Duração (média \pm EP) (dias) dos estágios de lagarta, pupa e do período de lagarta à emergência do adulto de Grapholita molesta em duas dietas artificiais.

\begin{tabular}{|c|c|c|c|c|c|c|}
\hline \multirow{2}{*}{ Dieta } & \multicolumn{2}{|c|}{ Lagarta } & \multicolumn{2}{|c|}{ Pupa } & \multicolumn{2}{|c|}{ Período (lagarta-adulto) } \\
\hline & Fêmeas & Machos & Fêmeas & Machos & Fêmeas & Machos \\
\hline Ivaldi-Sender & $\begin{array}{c}16,3 \pm 0,21^{* *} \\
(76)\end{array}$ & $\begin{array}{c}15,2 \pm 0,14^{* *} \\
(64)\end{array}$ & $\begin{array}{c}9,6 \pm 0,17^{* *} \\
(69)\end{array}$ & $\begin{array}{c}9,3 \pm 0,18^{* *} \\
(60)\end{array}$ & $\begin{array}{c}29,2 \pm 0,27^{* *} \\
(69)\end{array}$ & $\begin{array}{c}27,9 \pm 0,22^{* *} \\
(60)\end{array}$ \\
\hline À base de milho & $\begin{array}{c}19,5 \pm 0,24 \\
(60)\end{array}$ & $\begin{array}{c}18,9 \pm 0,23 \\
(83)\end{array}$ & $\begin{array}{c}8,5 \pm 0,15 \\
(54)\end{array}$ & $\begin{array}{c}8,7 \pm 0,12 \\
(75)\end{array}$ & $\begin{array}{c}31,7 \pm 0,24 \\
(54)\end{array}$ & $\begin{array}{c}31,0 \pm 0,25 \\
(75)\end{array}$ \\
\hline
\end{tabular}

Valores médios diferem $\left({ }^{* *}\right.$ ) entre dietas (na coluna) pelo teste $T$ com $P<0.05$. Valores entre parênteses expressam o número de observações.

Estes resultados coincidem com aqueles observados por Rosenthal et al. (1994) quando testaram, para a mariposa-oriental, a dieta de Guennelon, proposta por Guennelon et al. (1981) para a criação de Cydia pomonela (Linnaeus)
(Lepidoptera: Tortricidae). Rosenthal et al. (1994) verificaram que a presença de $5 \%$ de maçã seca triturada na dieta de Guennelon também reduziu o tempo de desenvolvimento das lagartas de $G$. molesta. 
ARIOLI, C. J. et al. Biologia da mariposa oriental em dieta...

TABELA 3 - Duração dos períodos de pré-oviposição, oviposição e embrionário (média \pm EP) (dias) e viabilidade embrionária (\%) de Grapholita molesta em duas dietas artificiais.

\begin{tabular}{ccccc}
\hline \multirow{2}{*}{ Dieta } & Pré-oviposição & Oviposição & \multicolumn{2}{c}{ Período embrionário } \\
\cline { 3 - 5 } & & & Duração (dias) & Viabilidade (\%) \\
\hline Ivaldi-Sender & $2,7 \pm 0,32^{\text {n.s }}$ & $24,9 \pm 0,94 * *$ & $4,38 \pm 0,07^{\text {n.s }}$ & $97,8 \pm 1,30^{\text {n.s }}$ \\
& $(20)$ & $(20)$ & $(822)$ & $(822)$ \\
À base de milho & $2,6 \pm 0,18$ & $19,1 \pm 1,67$ & $4,22 \pm 0,14$ & $99,0 \pm 0,31$ \\
& $(20)$ & $(20)$ & $(1023)$ & $(1023)$ \\
\hline
\end{tabular}

Valores médios diferem ${ }^{* *}$ ) e não diferem (n.s) entre dietas (na coluna) pelo teste $T$ com $P<0.05$. Valores entre parênteses expressam o número de observações.

TABELA 4 - Longevidade de adultos (dias) e fecundidade diária (ovos/fêmea/dia) e total (ovos/fêmea) (médiatEP) de Grapholita molesta em duas dietas artificiais.

\begin{tabular}{ccccc}
\hline \multirow{2}{*}{ Dieta } & \multicolumn{2}{c}{ Longevidade $(20)$} & \multicolumn{2}{c}{ Fecundidade (20) } \\
\cline { 2 - 4 } & Fêmeas & Machos & Diária & Total \\
\hline Ivaldi-Sender & $28,7 \pm 1,04^{* *}$ & $26,6 \pm 2,34^{* *}$ & $13,7 \pm 2,97^{\text {ns }}$ & $333,8 \pm 11,47^{* *}$ \\
À base de milho & $23,2 \pm 1,62$ & $20,9 \pm 0,95$ & $13,5 \pm 1,45$ & $234,8 \pm 18,95$ \\
\hline
\end{tabular}

Valores médios diferem ( ${ }^{* *}$ ) e não diferem (n.s) entre dietas (na coluna) pelo teste $\mathrm{T}$ com $\mathrm{P}<0.05$

De forma geral, os valores obtidos para a duração dos estágios imaturos, com insetos alimentados com dieta à base de milho, comparamse aos obtidos quando indivíduos da mesma espécie são criados em dietas artificiais e naturais, respectivamente (Rosenthal et al., 1994).

Embora a dieta a base de milho tenha originado insetos com menor massa de pupa para ambos os sexos (Tabela 5), os valores são superiores aos obtidos por outros autores que criaram G. molesta em dietas artificiais. Tzanakakis \& Phillips (1969) usando dietas artificiais à base de folhas de alfafa e cenoura, obtiveram valores não superiores a $10,2 \mathrm{mg}$ para machos e a $11,9 \mathrm{mg}$ para fêmeas, respectivamente. Para Szocs \& Tóth (1982) e Yokoyama et al.(1987), que utilizaram na mistura da dieta germe de trigo e feijão-de-lima respectivamente, a média de massa das pupas de machos não ultrapassou $11,4 \mathrm{mg}$. Também para as fêmeas, as dietas não originaram pupas com massa acima de 15,3 mg. Além disso, o massa de pupa observado na dieta à base de milho foi maior que os observados por Rosenthal et al. (1994), quando criaram a mariposa-oriental nas dietas artificiais de Ivaldi-Sender, de Guennelon e com frutos de maçã e marmelo.

TABELA 5 - Viabilidade (\%) dos estágios de lagarta, pupa e do período de lagarta à emergência do adulto (lagarta-adulto) e massa de pupas (mg) (média \pm EP) de Grapholita molesta em duas dietas artificiais.

\begin{tabular}{lccccc}
\hline \multirow{2}{*}{ Dieta } & \multicolumn{2}{c}{ Viabilidade dos estágios (\%) } & \multicolumn{2}{c}{ Massa de pupas (mg) } \\
\cline { 2 - 6 } & Lagarta & Pupa & Lagarta-adulto & Machos & Fêmeas \\
\hline \multirow{2}{*}{ Ivaldi-Sender } & $\begin{array}{c}98,0 \pm 1,15^{\text {n.s }} \\
(150)\end{array}$ & $\begin{array}{c}89,1 \pm 2,58^{\text {n.s }} \\
(147)\end{array}$ & $\begin{array}{c}87,3 \pm 2,72^{\text {n.s }} \\
(150)\end{array}$ & $\begin{array}{c}18,3 \pm 0,32^{* *} \\
(76)\end{array}$ & $\begin{array}{c}13,8 \pm 0,25^{* *} \\
(64)\end{array}$ \\
& & & & \\
À base de milho & $\begin{array}{c}95,3 \pm 1,73 \\
(150)\end{array}$ & $\begin{array}{c}90,9 \pm 2,41 \\
(143)\end{array}$ & $\begin{array}{c}87,2 \pm 2,74 \\
(150)\end{array}$ & $\begin{array}{c}15,7 \pm 0,43 \\
(60)\end{array}$ & $\begin{array}{c}13,1 \pm 0,23 \\
(82)\end{array}$ \\
\hline
\end{tabular}

Valores médios diferem $\left({ }^{* *}\right)$ e não diferem (n.s) entre dietas (na coluna) pelo teste T com P<0.05. Valores entre parênteses expressam o número de observações.

A viabilidade das fases de lagarta, de pupa e de lagarta-adulto não foi alterada pelas dietas (Tabela 5). No entanto, os valores obtidos para o estágio de lagarta foram superiores aos verificados Grellmann et al. (1991) usando dietas artificiais e aos de Rosenthal et al. (1994) com dietas naturais.
A viabilidade pupal também foi maior ou semelhante às observadas por autores que criaram G. molesta com dieta artificial (Ivaldi-Sender, 1974; Szocs \& Tóth, 1982; Yokoyama et al,1987; Rosenthal et al.,1994). 
ARIOLI, C. J. et al. Biologia da mariposa oriental em dieta...

De acordo com Tzanakakis \& Phillips (1969) e Yokoyama et al. (1987), a condição física da dieta constitui-se em fator crítico para o estabelecimento e sobrevivência de lagartas da mariposa-oriental recém-eclodidas. Dessa maneira, tal condição foi adequadamente mantida na dieta a base de milho, fato observado pela alta viabilidade (> 95\%) da fase de lagarta (Tabela 5).

Observou-se um desvio na proporção de sexos, sendo o número de fêmeas menor na dieta à base de milho ( $r s=0,42$ ), ao contrário do observado na dieta de Ivaldi-Sender, onde mais fêmeas foram encontradas ( $r s=0,54)$. A razão sexual na dieta à base de milho assemelha-se àquela obtida por Rosenthal et al. (1994), que encontraram uma razão sexual entre 0,45 e 0,49 ao criarem G. molesta em dieta natural (marmelo) e artificial (Guennelon + maçã), respectivamente. Rosenthal et al. (1994) também verificaram que a dieta de Ivaldi-Sender dá origem a um maior número de fêmeas, corroborando o resultado anteriormente apresentado.

Não houve diferença no período de préoviposição entre as dietas testadas (Tabela 3). Já o período de oviposição foi significativamente maior, com 24,9 dias na dieta de Ivaldi-Sender contra 19,1 dias na dieta à base de milho.

Com relação à longevidade, tanto machos quanto fêmeas alimentados na fase de lagarta com a dieta de Ivaldi-Sender viveram mais que os machos e fêmeas alimentados na dieta à base de milho (Tabela 4). Mesmo assim, a eliminação do componente maçã seca e o acréscimo de milho à dieta à base de milho revelou que tanto os machos quanto as fêmeas viveram mais que aqueles criados em dieta natural (maçãs verdes armazenadas) (Marin et al., 2006). Em hipótese, alguns autores sugerem que G. molesta criada com maçãs verdes armazenadas fica desnutrida, fato atribuído à perda nutricional que ocorre nos frutos quando armazenados por longo período de tempo (Tzanakakis \& Phillips, 1969; Szocs \& Tóth, 1982). De maneira geral, há concordância com as pesquisas de Panizzi \& Parra (1991), que alegam que as diferenças encontradas na longevidade dos adultos têm relação direta com a qualidade do alimento ingerido pelas lagartas.

Não houve diferença significativa na fecundidade diária entre as duas dietas testadas (Tabela 4). Já a fecundidade total de G. molesta foi significativamente superior para a dieta de IvaldiSender (Tabela 4). Para as duas dietas, os resultados são superiores aos observados na literatura. Tzanakakis e Phillips (1969) obtiveram apenas 126 ovos/fêmea quando as lagartas foram criadas com maçã verde e 124 ovos/fêmea na dieta à base de alfafa e cenoura. Yokoyama et al. (1987) observaram 100 ovos/fêmea na dieta formulada com feijão-de-lima e Alfonso \& Marin (2004) obtiveram 143 e 131 ovos/fêmea quando as lagartas foram mantidas em laboratório em maçãs verdes e em dieta artificial respectivamente, enquanto que, em outro trabalho, Marín et al. (2006) obtiveram 160 ovos/fêmea com dieta de maçãs verdes.

Não foi observada diferença significativa no período embrionário e na viabilidade dos ovos de G. molesta criada nas duas dietas (Tabela 3). Para a viabilidade dos ovos, os valores assemelham-se aos encontrados por Alfonso \& Marín (2004) criando lagartas de G. molesta em dieta natural e artificial. No entanto, divergem daqueles alcançados por Rosenthal et al. (1994), os quais, estudando diferentes dietas artificiais e naturais, verificaram uma viabilidade máxima de $53,5 \%$, apenas quando acrescentaram $1 \%$ de sais de Wesson à dieta proposta por Ivaldi-Sender. Estas diferenças podem estar relacionadas ao tipo de gaiola utilizada para os estudos da fecundidade da mariposa-oriental, já que Peters \& Barbosa (1977) argumentam que sempre existe um espaço físico ótimo para cada fase de desenvolvimento dos insetos

A duração média de uma geração da mariposa-oriental, calculada através da tabela de vida de fertilidade, variou entre 37,78 dias na dieta de Ivaldi-Sender e 39,22 dias na dieta à base de milho (Tabela 6). O número de vezes que a população aumenta a cada geração (taxa líquida de reprodução $=$ Ro) foi menor em 1,87 vez quando o inseto foi criado na dieta à base de milho (Tabela 6 ). Os valores de capacidade inata de aumentar em número (razão infinitesimal de aumento $=\mathrm{rm}$ ) e a razão finita de aumento $(\lambda)$, que representa o número de fêmeas adicionadas à população por fêmea numa unidade de tempo, foram maiores na dieta de Ivaldi-Sender (Tabela 6).

TABELA 6 - Duração média de uma geração (T), taxa líquida de reprodução (Ro), capacidade inata de aumentar em número $(\mathrm{rm})$ e razão finita de aumento $(\lambda)$ de Grapholita molesta em duas dietas artificiais.

\begin{tabular}{|c|c|c|c|c|}
\hline Dieta & $\mathrm{T}$ (dias) & Ro & $\mathrm{Rm}$ & (I) \\
\hline Ivaldi-Sender & 37,78 & 3092,06 & 0,212715 & 1,23703 \\
\hline À base de milho & 39,22 & 1639,15 & 0,188729 & 1,20800 \\
\hline $\begin{array}{l}\text { Consideran } \\
\text { fertilidade como um } \\
\text { de uma dieta par } \\
\text { observou-se que a c } \\
\text { a que apresento } \\
\text { proporcionou a men }\end{array}$ & $\begin{array}{l}\text { tabela d } \\
\text { avaliação } \\
\text { ção de } \\
\text { a de Ivald } \\
\text { desemp } \\
\text { o de dese }\end{array}$ & & \multicolumn{2}{|c|}{$\begin{array}{l}\text { e maior razão finita de aumento (Tabela } 6 \text { ). Mesmo } \\
\text { assim, a dieta proposta à base de milho, mostrou-se } \\
\text { adequada à criação de G. molesta, pois } \\
\text { proporcionou uma viabilidade total do período } \\
\text { lagarta-adulto da ordem de } 87,2 \% \text { (Tabela } 4 \text { ), valor } \\
\text { superior aos } 75 \% \text { preconizados por Singh (1983) }\end{array}$} \\
\hline
\end{tabular}


ARIOLI, C. J. et al. Biologia da mariposa oriental em dieta...

como referencial para que uma dieta seja considerada adequada para a criação contínua de inseto em laboratório.

Os resultados ora apresentados aliados ao pequeno percentual de pupas $(4,9 \%)$ e adultos $(0 \%)$ deformados indicam que a dieta à base de milho também é adequada à criação de G. molesta. Dessa forma, mesmo com componentes diferentes dos constituintes da dieta natural (frutos de maçã, pêssego e marmelo, por exemplo), a dieta à base de milho, que tem sido utilizada atualmente em muitos laboratórios do Brasil, pode ser utilizada para a criação de G. molesta.

\section{CONCLUSÕES}

Os resultados do estudo indicam que:

a) Lagartas de G. molesta criadas na dieta à base de milho apresentam maior duração do período lagarta-adulto:

b) G. molesta criada na dieta à base de milho apresenta menores massas de pupa, período de oviposição, longevidade de adultos (machos e fêmeas) e fecundidade total;

c) Lagartas de G. molesta criadas na dieta à base de milho apresentam uma viabilidade total do período lagarta-adulto da ordem de $87,2 \%$;

d) A dieta à base de milho é adequada à criação de G. molesta em laboratório.

\section{REFERÊNCIAS}

1. ALFONSO, A. M.; MARÍN, M. S. Grapholita molesta en condiciones de laboratorio: Evaluación de la relación de sexos. Revista de la Facultad de Ciencias Agrarias, v. 36, n. 2, p. 23-29, 2004.

2. ARIOLI, J. C. et al. Bioecologia e controle de Grapholita molesta (Busck,1916) (Lepidoptera: Tortricidae) em fruteiras temperadas. In: ENCONTRO NACIONAL SOBRE FRUTICULTURA DE CLIMA TEMPERADO, 6., 2003, Fraiburgo, SC. Anais... Caçador: EPAGRI, 2003, p. 152-162.

3. ARIOLI, J. C.; BOTTON, M.; CARVALHO, G. A. C. Controle químico de Grapholita molesta (Busck) (Lepidoptera:Tortricidae) na cultura do pessegueiro. Ciência Rural, v. 34, n. 6, p.1695-1700, 2004.

4. ARIOLI, J. C. et al. Técnica de criação de Grapholita molesta (Busck, 1916) (Lepidoptera: Tortricidae) em laboratório utilizando dieta artificial para produção de insetos visando estudos de comportamento e controle. Bento Gonçalves: Embrapa Uva e Vinho, 2007. 14 p. (Boletim de pesquisa e desenvolvimento, 13).

5. BEEKE, H.; JONG, D. de Identification of larvae and pupa. In: GREEST, L. P. S. V.; EVENHUIS, H. H. (Eds.). World crop pests: Tortricids pests: their biology, natural enemies and control. Amsterdam: Elsevier, 1991. p. 65-71.

6. GRELLMANN, E. O et al. Ciclo evolutivo de Grapholita molesta (Busck,1916) (Lepidoptera: Olethreutidae) em diferentes temperaturas. Revista Brasileira de Fruticultura, v. 13, n. 4, p. 21-26, 1991.

7. GUENNELON, G et al. Progrès réalisés dans l'élevege permanent du Carpocapse (Laspeyresia pomonella L.) sur milieu artificiel. Agronomie, v. 1, n. 1, p. 59-64, 1981.

8. IVALDI-SENDER, C. Techniques simples pour un élevage permanent de la Tordeuse Orientale, Grapholita molesta (Lepidoptera: Tortricidae) sur milieu artificiel. Annales Zoologie Ecologie Animale, v. 6, n. 2, p. 337-343, 1974.

9. KOVALESKI, A.; RIBEIRO,L.G. Manejo de Pragas na Produção Integrada de maçã. In: PROTAS, J.F.S.; VALDEBENITO SANHUEZA, R.M. (Eds.). Produção integrada de frutas: o caso da maçã no Brasil. Bento Gonçalves: Embrapa Uva e Vinho, 2003. p. 61-68.

10. MARÍN, M. S et al. Grapholita molesta: Caracterización de uma cria artificial. Revista de la Facultad de Ciencias Agrarias, v. 38, n. 1, p. 7-12, 2006.

11. MONTEIRO, L. B.; HICKEL, E. Pragas de importância econômica em fruteiras de caroço. In: MONTEIRO, L. B. et al (Ed.) Fruteiras de caroço: uma visão ecológica. Curitiba, UFPR, 2004. p. 223-264.

12. NORA, I.; HICKEL, E. R. Pragas da macieira. In: EPAGRI. Manual da cultura da macieira. Florianópolis, 2002, p. 463525 .

13. NORA, I.; SUGIURA, T. Pragas da pereira. In: EPAGRI (Ed.) Nashi: a pêra japonesa. Florianópolis: EPAGRI: JICA 2001. p. 261-321.

14. PANIZZI, A. R.; PARRA, J. R. P. Ecologia nutricional de insetos e suas implicações no manejo de pragas. São Paulo: MALONE, 1991. 359 p.

15. PETERS, T. M.; BARBOSA, P. Influence of population density on size, fecundity, and developmental rate of insects in culture. Annual Review of Entomology, v. 22, n. 2 p. 431-450, 1977.

16. RIBEIRO JUNIOR, J. I. Análises estatísticas no SAEG. Viçosa: UFV, 2001. $301 \mathrm{p}$.

17. RIBBERT, D. A.; MARÍN, M. S. Densidad de adultos de Grapholita molesta en jaulas. Revista de la Facultad de Ciencias Agrarias, v. 36, n. 1, p. 25-30, 2005.

18. ROSENTHAL, M. de. A.; LOECK, A. E.; SILVA JUNIOR, P. Criação de Grapholita molesta (Busck, 1916) (Lepidoptera: Olethreutidae) em dietas artificiais e naturais. Pesquisa Agropecuária Brasileira, v. 29, n. 12, p.1847-1853, 1994.

19. SALLES, L. A. B. de. A Mariposa-oriental, Grapholita molesta (Lepidoptera: Tortricidae). In: VILELA, E. F.; ZUCCHI, R A.; CANTOR, F. (Ed.). Histórico e impacto das pragas introduzidas no Brasil. Ribeirão Preto: Holos, 2001. p. 42-45.

20. SZOCS, G.; TÓTH, M. Rearing of the Oriental Fruit Moth, Grapholita molesta Busck, on simple semi synthetic diets. Acta Phytopathologica Academiae Scientiarum Hungaricae, v. 17, n. 3-4, p. 295-299, 1982.

21. SILVEIRA NETO, S et al. Manual de ecologia dos insetos. São Paulo: Agronômica Ceres, 1976. 419 p.

22. SINGH, P. A General purpose laboratory diet mixture for rearing insects. Insect Science and its Applications, v. 4, n. 4 p. 357-362, 1983.

23. TZANAKAKIS, M. E.; PHILLIPS, J. H. H. Artificial diets for larvae of the Oriental Fruit Moth. Journal of Economic Entomology, v. 62, n. 4, p. 879-882, 1969

24. YOKOYAMA, V. Y.; MILLER, G. T.; HARVEY, J. M. Development of the oriental fruit moth (Lepidoptera: Tortricidae) on a laboratory diet. Journal of Economic Entomology, v. 80, n. 1, p. 272-276, 1987.

Recebido em 26/02/2010 Aceito em 16/12/2010 